\title{
Multi-modal imaging of the subscapularis muscle
}

\author{
Mona Alilet $^{1}$ • Julien Behr ${ }^{2}$ • Jean-Philippe Nueffer ${ }^{1}$ - Benoit Barbier-Brion ${ }^{3}$. \\ Sébastien Aubry ${ }^{1,4}$
}

Received: 31 May 2016/Revised: 6 September 2016/Accepted: 28 September 2016/Published online: 17 October 2016

(C) The Author(s) 2016. This article is published with open access at Springerlink.com

\begin{abstract}
The subscapularis (SSC) muscle is the most powerful of the rotator cuff muscles, and plays an important role in shoulder motion and stabilization. SSC tendon tear is quite uncommon, compared to the supraspinatus (SSP) tendon, and, most of the time, part of a large rupture of the rotator cuff. Various complementary imaging techniques can be used to obtain an accurate diagnosis of SSC tendon lesions, as well as their extension and muscular impact. Pre-operative diagnosis by imaging is a key issue, since a lesion of the SSC tendon impacts on treatment, surgical approach, and postoperative functional prognosis of rotator cuff injuries. Radiologists should be aware of the SSC anatomy, variability in radiological presentation of muscle or tendon injury, and particular mechanisms that may lead to a SSC injury, such as coracoid impingement.
\end{abstract}

\section{Teaching Points}

- Isolated subscapularis (SSC) tendon tears are uncommon.

- Classically, partial thickness SSC tendon tears start superomedially and progress inferolaterally.

Sébastien Aubry

radio.aubry@free.fr

1 Department of Musculoskeletal Imaging, CHRU de Besançon, CHRU Jean Minjoz, Boulevard Fleming, 25030 Besançon Cedex, France

2 Anatomy Laboratory, University of Franche-Comte, Besançon, France

3 Groupe CIMRAD, Centre d'imagerie des Tilleroyes, Besançon, France

4 Nanomedicine and Imagery Laboratory, EA4662, University of Franche-Comte, Besançon, France
- Long head of biceps tendon medial dislocation can indirectly signify SSC tendon tears.

- SSC tendon injury is associated with anterior shoulder instability.

- Dynamic ultrasound study of the SSC helps to diagnose coracoid impingement.

Keywords Subscapularis · Tendon injury · Rotator cuff · Magnetic resonance imaging · Coracoid impingement

\section{Introduction}

The subscapularis (SSC) muscle is one of the four components of the rotator cuff along with the supraspinatus (SSP), infraspinatus, and teres minor muscles. The long head of biceps (LHB) tendon is classically associated.

The muscles of the rotator cuff and their tendinous insertions play an important role in shoulder stability, and in the motion of the glenohumeral joint. The SSC in particular has the greatest force-producing capacity, followed by the infraspinatus, SSP, and teres minor [1].

Rotator cuff pathologies are very common, and foremost among these are SSP tendon tears. SSC tendon pathology is quite uncommon, and most of the time, occurs as part of a larger rupture of the rotator cuff.

In cadaveric studies, the prevalence of SSC tendon tears varies between 29 and $37 \%$ [2], whereas it is estimated to be between 5 and $27 \%$ in clinical studies [3, 4]. This discrepancy could be related to the incomplete visualization of the SSC tendon on both arthroscopy and open surgery [5].

Pre-operative diagnosis of SSC tendon tears is therefore a major issue, especially since a lesion of the SSC tendon will have an impact on treatment, surgical approach, and postoperative functional prognosis [6]. The purposes of this 
article are to describe the normal appearance of the SSC, and to review the imaging findings encountered in patients with lesions of the SSC tendon and muscle, according to specific pathophysiological mechanisms.

\section{Anatomy}

The SSC muscle is the largest and the most powerful of the rotator cuff muscles. It is located in the subscapular fossa at the anterior aspect of the scapula. It contains multiple tendinous and muscular bundles that merge laterally into a flattened tendon in the upper two-thirds of the muscle. This tendinous portion has a variable insertion, mostly on the lesser tuberosity of the humerus, but also on the greater tuberosity and the bicipital groove [2]. SSC tendon fibres contained within a fibrous expansion from the pectoralis major tendon form the transverse ligament, which is a fascia covering the vertical portion of the LHBT (Fig. 1).

The lower third of the muscle has a muscular attachment onto the inferior part of the lesser tuberosity and onto the anterior aspect of the humeral diaphysis.

\section{Innervation}

Variants of the innervation of the SSC muscle are common [7]. Classically, the two superior thirds and the inferior third of the SSC muscle are innervated, respectively, by the upper and lower subscapularis nerves, which are both branches of the posterior chord of the brachial plexus (Fig. 1). Electromyographic (EMG) studies have shown differences in activity between the upper and lower portion of the SSC muscle, suggesting that they work as two different muscular units during voluntary shoulder movements [8].

\section{Function}

The principal role of the SSC muscle in shoulder motion is internal rotation. In variable positions, the superior subscapularis fibres assist in abduction, while the inferior fibres assist in adduction. At the beginning of elevation shoulder movement, EMG onset of the upper portion of the SSC occurs first, which increases the glenohumeral congruence. The upper SSC also demonstrates higher levels of activation than the lower portion, which confirms the hypothesis of a participation in abduction [8].

The SSC muscle provides active stabilization of the shoulder during external rotation and flexion [9]. Its action is coordinated with the other muscles of the rotator cuff to ensure shoulder function and the centring of the humeral head in the glenoid fossa. There are balanced forces between the superior fibres of the SSC and its antagonist, the infraspinatus muscle, in the axial plane. Conversely, the inferior fibres of the SSC resist, in the coronal plane, the elevation of the humeral head induced by the deltoid muscle [2].

The SSC muscle also plays a role in passive stability of the glenohumeral joint, resisting anterior luxation. Indeed, anterior luxation is associated with laxity of the lower portion of the SSC muscle [10]. This explains why some authors recommend surgical repair of the SSC for treatment of anterior glenohumeral instability [11]. Also, the SSC tendon stabilizes the LHBT within the rotator interval (see below).

\section{Anatomical relations}

\section{Rotator interval}

The SSC tendon contributes to the formation of an anatomical space located in the anterosuperior portion of the shoulder, called the rotator interval (Fig. 2), which is a tendinous gap in
Fig. 1 Anatomical views of the shoulder: front view (a), lateral oblique view (b). 1: Subscapularis muscle. 2: Subscapularis tendon. 3: long head of biceps tendon. 4: Coracoid process. 5: Teres major muscle. 6: Coraco-brachialis muscle. 7: Brachial plexus. 8: Lower subscapularis nerve. 9: Axillary artery a

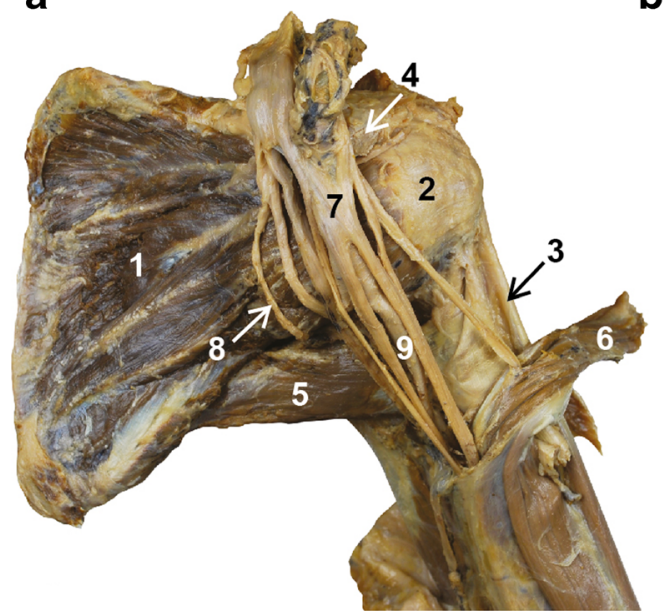

b

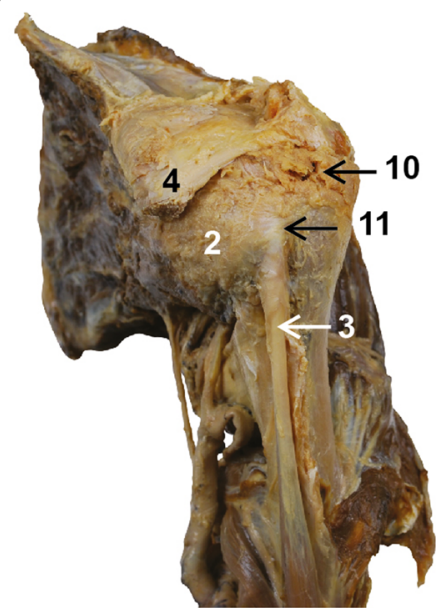


Fig. 2 Schematic representation of the rotator interval (RI): front view (a), sagittal view (b). The RI is bordered by the SSC tendon inferiorly (1) and the SSP tendon superiorly (2) and contains the intra-articular portion of LHBT (3). CHL (4) and SGHL (5) surround the LHBT in the RI, acting as a pulley system for LHBT stabilization

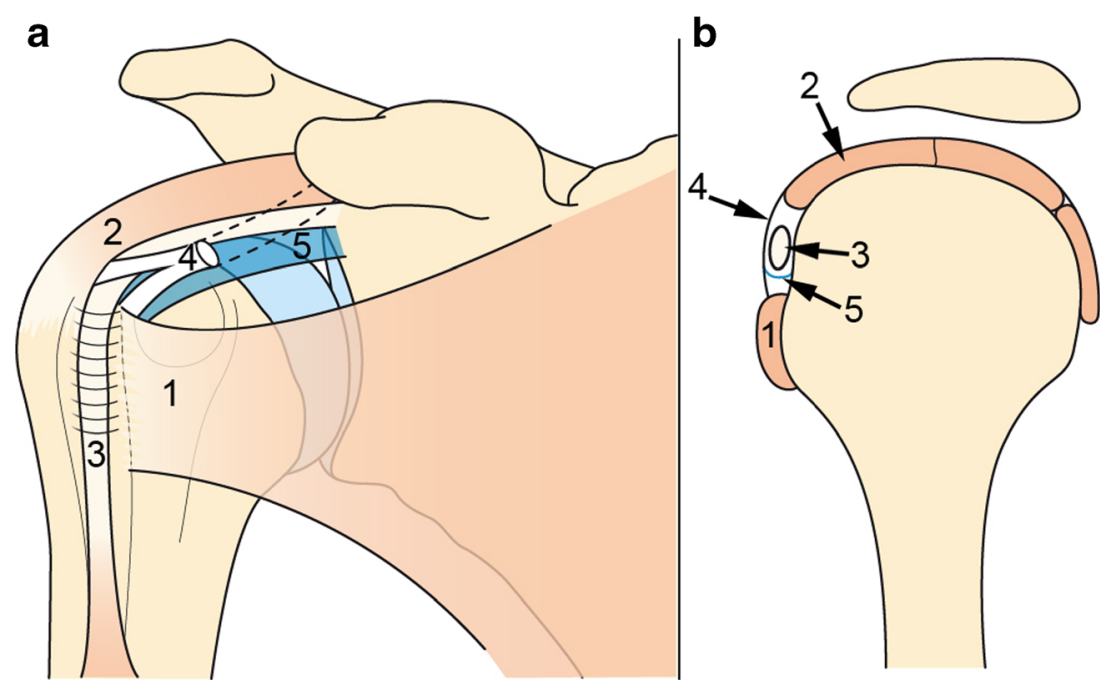

the rotator cuff, exclusively covered by capsular tissue. It is a triangular shaped space bordered inferiorly by the superior free edge of the SSC tendon, and superiorly by the anterior free edge of the SSP tendon. The base of this triangle is formed by the coracoid process medially, and its apex is the intertubercular sulcus laterally [12]. The anterior aspect of the rotator interval is formed by a fibrous capsule made of blended fibres coming from the SSC and SSP tendons. It is reinforced by two ligaments, namely the coracohumeral ligament (CHL) and the superior glenohumeral ligament (SGHL). The inferomedial CHL, the SGHL and the superior fibres of the SSC tendon unite in the lateral rotator interval and act as a pulley system for the LHBT, which is a key element for stabilization of the LHBT [13]. Indeed, this pulley system prevents inferior luxation of the horizontal portion of the LHBT, and medial luxation of its vertical portion. The most superior insertion point of the SSC could be the most important structure for medial stabilization of LHBT [14].
Fig. 3 B-mode ultrasound images of the SSC. a Transverse view (long axis) : the normal SSC tendon is hyperechoic compared to the adjacent muscle and has a fibrillary structure. b Sagittal view (short axis): multi-pennate structure of the SSC tendon with alternation of hypo and hyperechoic zones
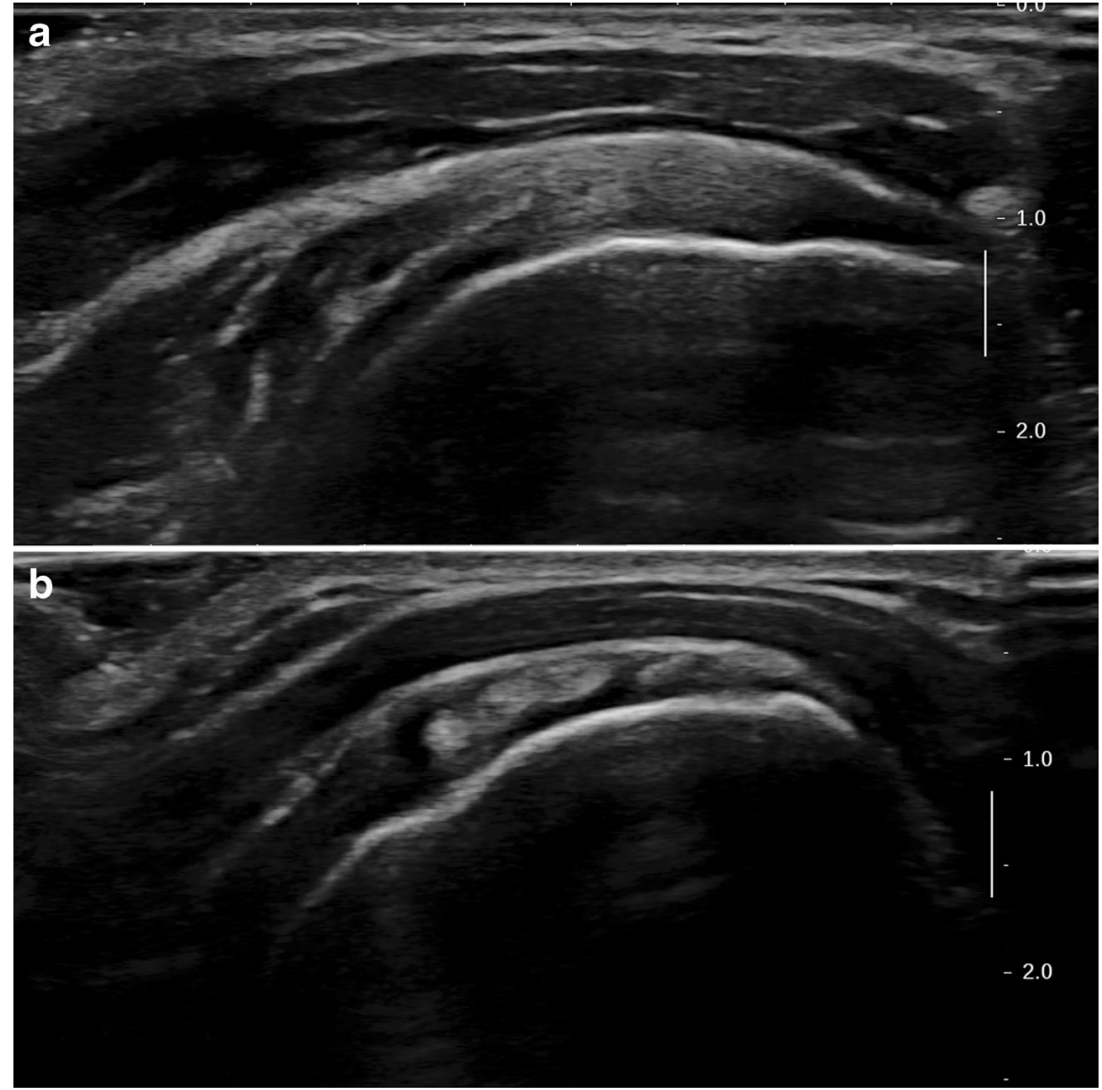
Fig. 4 Calcific tendinitis of the SSC. Calcificationsof the SSC tendon (white arrows) overlap the lesser tuberosity in the front view in neutral rotation (a), are lateralized in external rotation (b), and medialized in internal rotation (c). On the Y view, calcifications of SSC tendon are seen under the coracoid process (white arrowhead) (d)
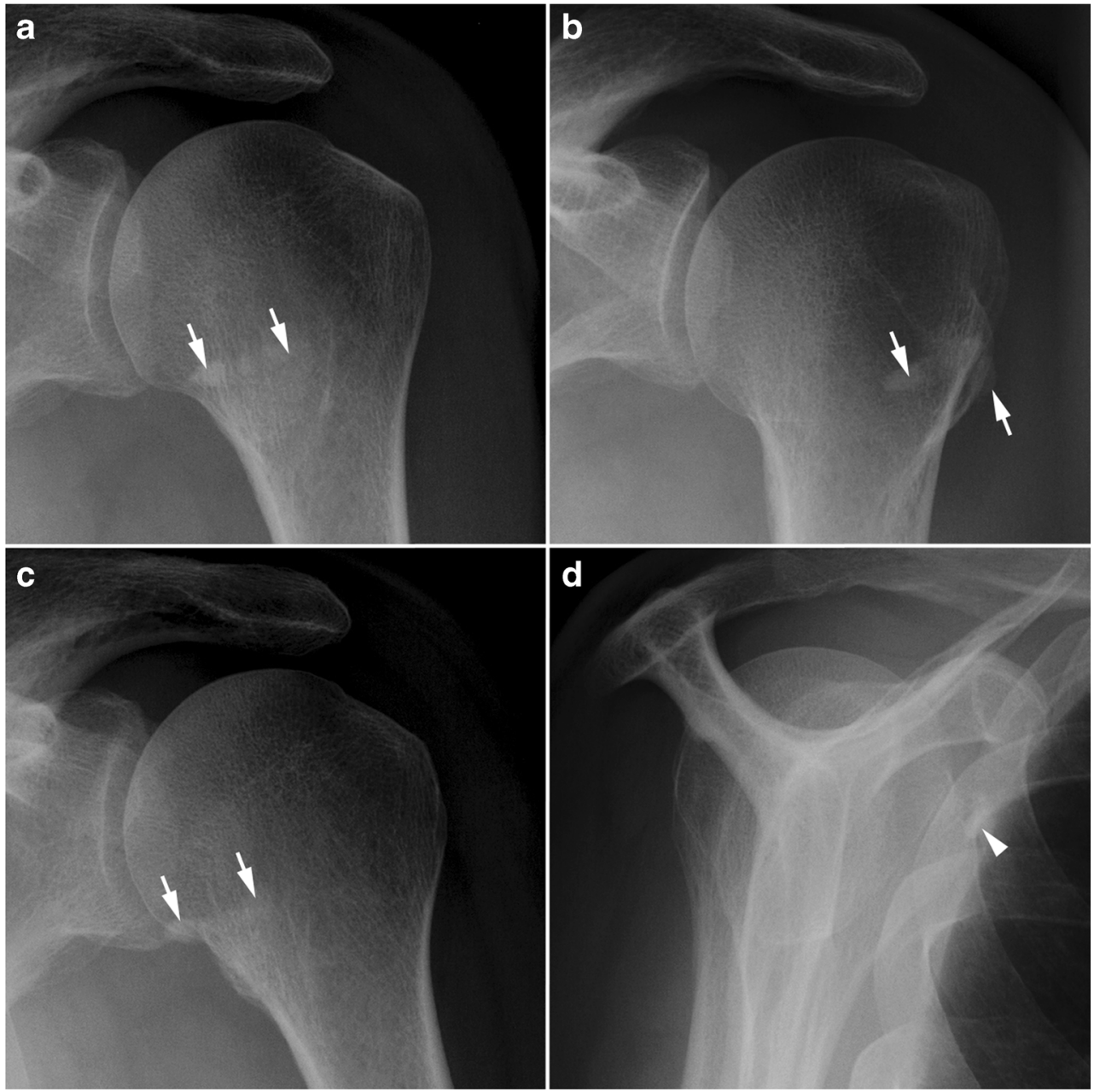

\section{Other anatomical relations}

The origin of the vascular-nervous pedicle of the upper limb is located at the anterior aspect of the SSC muscle. The axillary artery and vein, and the chords of the brachial plexus lean on the SSC muscle. In front of the SSC muscle, the medial, lateral and posterior chords of the brachial plexus divide into their terminal branches (Fig. 1).

Among the anatomical variations of the shoulder, an accessory coracobrachialis muscle that crosses in front of the SSC tendon is observed in $1 \%$ of cases [15].

Also, in the coronal plane there is a continuity between the SSS muscle, inserted in the subscapularis fossa, and the teres major, inserted on the lateral aspect of the scapula (Fig. 1).

\section{Clinical features}

Most rotator cuff tears are asymptomatic [16]. When a SSC tendon lesion is symptomatic, spontaneous or triggered pain is preferentially located in the anterior region of the shoulder. A tear is painful during development, whereas a constituted tear is less painful, if at all. When subacromial-subdeltoid (SASD) bursitis is associated, pain is common. Calcific tendinitis can also be painful, especially when hydroxyapatite calcifications are in the resorptive phase [17].

Most of the time, clinical examination can orientate towards an articular or periarticular pathology, but it cannot identify the precise topography or the pathological mechanism, even if there are many clinical tests [18]. The SSC tendon is evaluated by Gerber's "lift-off test" [19], or the "Belly press test". It can also be tested by evaluating resisted internal rotation at maximal abduction $\left(90^{\circ}\right)$ or maximal external rotation [20]. Finally, when the SSC tendon is torn, external rotation of the shoulder will be increased in passive mobilization [19].

\section{Pathogenesis of SSC tendon lesions}

Several factors can concur to weaken the SSC tendon. Rarely, in young patients, SSC tears can occur on a 


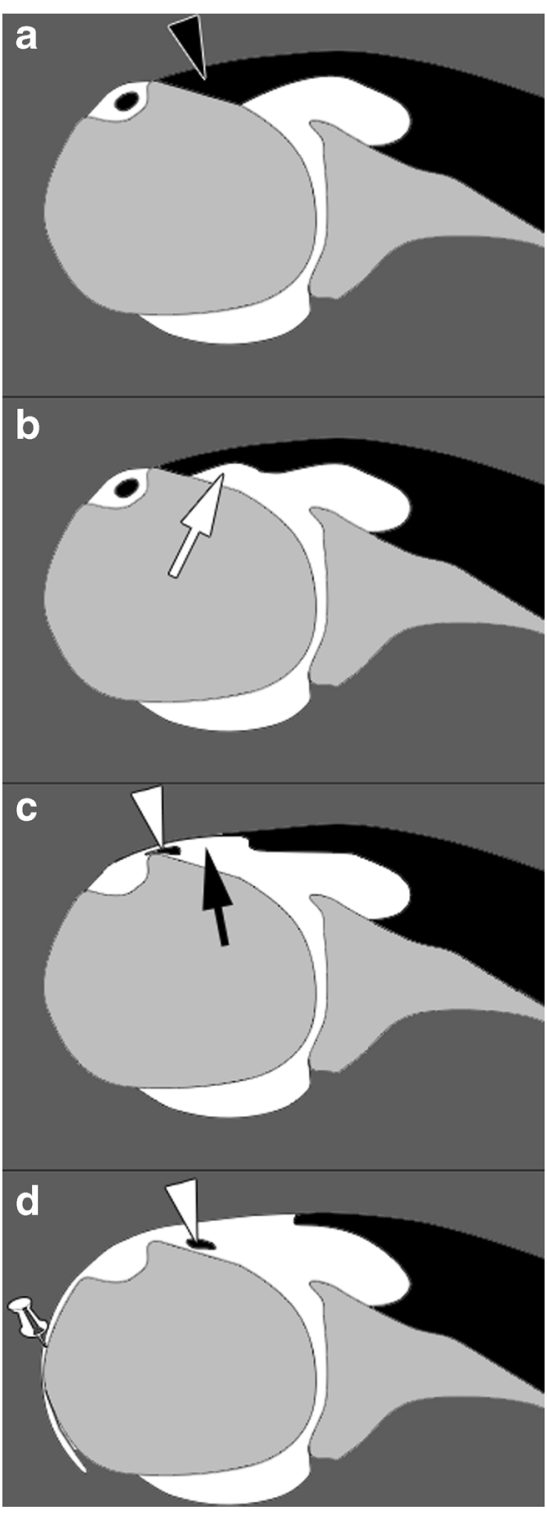

Fig. 5 Schematic representation of SSC tendon tears on magnetic resonance or computed tomography arthography. Normal SSC tendon (black arrowhead) (a). Incomplete partial thickness tear (white arrow) (b). Complete partial thickness tear (black arrow) associated with LHBT (white arrowhead) subluxation (c). Complete full thickness tear associated with LHBT (white arrowhead) dislocation and opacification of the SASD bursa (pin) (d)

healthy tendon and result from kinetic trauma in traction. Frequently, SSC tendon tears occur on underlying chronic tendinopathy, which refers to tendinosis on histopathology. When these tendinosis phenomena are associated with calcium deposits within the substance of the tendon, this entity is called calcific tendinitis. Furthermore, extrinsic factors can generate excessive mechanical constraints on the SSC tendon. Among these, coracoid or anteromedial impingement and anterior instability of the shoulder are secondary to specific pathophysiological mechanisms that lead to the findings detailed below.

\section{Radioanatomy}

\section{Plain radiographs}

To explore the shoulder, anteroposterior views with three projections (neutral, external and internal rotation), an axillary view, and a scapular "Y" view must be performed. The axillary view can show alterations of the lesser tuberosity, such as irregularities or cysts, which argue in favour of a SSC tendon lesion [21]. Anterior subluxation of the humeral head can also be seen on this view [22]. The "Y" view is helpful to locate tendinous calcifications and to look for a narrow subcoracoid space, which can both lead to coracoid impingement (see below).

\section{Ultrasound}

The SSC tendon is first evaluated in its long axis, by placing the probe transversally over the anterior shoulder, in the same position as for the study of the LHBT. External rotation of the forearm, while keeping the elbow next to the chest, brings out the lesser tuberosity and SSC tendon laterally. The normal SSC tendon moves freely under the coracoid process and the LHBT must stay in its groove during external rotation. Next, a short axis view of the SSC tendon is obtained by turning the probe $90^{\circ}$, which demonstrates its multi-pennate structure (Fig. 3).

\section{MRI}

MRI images must be obtained with a dedicated shoulder coil. The shoulder is studied in three planes (i.e., axial, oblique coronal, oblique sagittal) with fat-suppressed (FS) fluid-sensitive sequences (proton density (PD) or T2 weighted). Axial and oblique sagittal planes are the most informative for the SSC tendon. The axial plane evaluates the SSC tendon in its long axis and makes it possible to explore the LHBT and its position within the intertubercular sulcus. The short axis of the SSC tendon and the multi-pennate structure of the SSC muscle are studied in the oblique sagittal plane.

An oblique sagittal T1-weighted sequence is also recommended to evaluate fatty infiltration and muscle mass.

\section{Magnetic resonance arthrography (MRA)}

An accurate and a traumatic shoulder arthrography technique is essential to avoid false images of SSC tendon lesions. The anterior approach targeting the rotator cuff interval under ultrasound or fluoroscopic guidance is currently the most widely performed technique [23], even if a posterior approach makes it possible to avoid any iatrogenic contamination of the SSC tendon or the SASD bursa. Ten to twelve cc of contrast medium (dilution of gadolinium at $0.0025 \mathrm{mmol} / \mathrm{L}$ ) are injected 
Fig. 6 Computed tomography arthrography of the left shoulder with axial (a) and sagittal oblique (b) multiplanar reconstructions in a 38-year-old man, in a posttraumatic context. Isolated complete partial thickness tear of the SSC tendon, located at the articular side of the tendon, involving the upper fibres (arrow head), responsible for subluxation of the LHBT (arrow). Absence of contrast in the SASD bursa (pin) confirms the absence of transfixing or full thickness tear

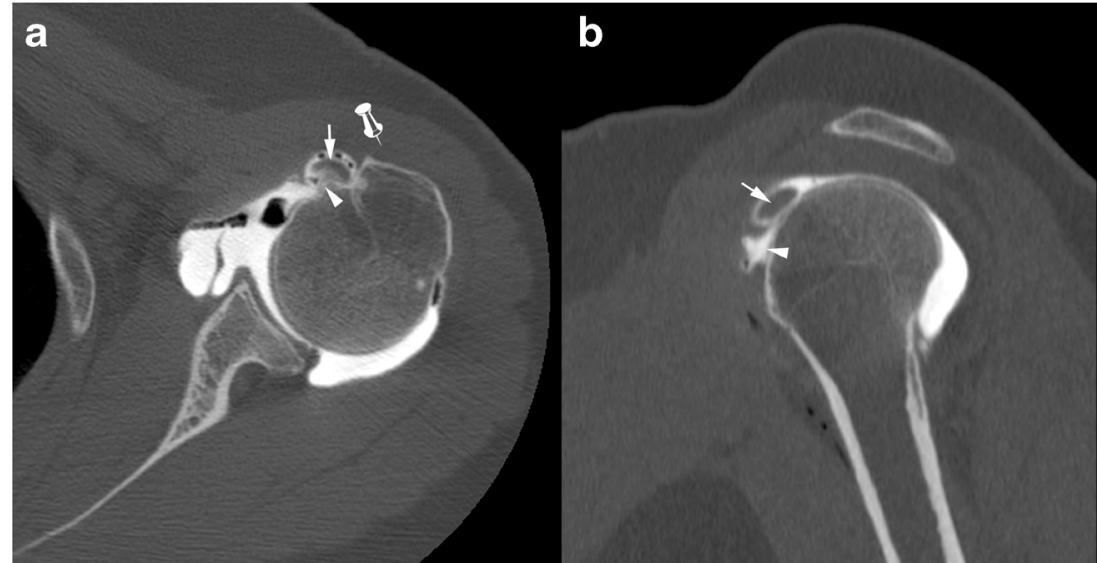

into the glenohumeral joint. The MRA acquisition protocol includes FS T1-weighted in three planes (or a 3DFS-T1 $1_{w}$ ), a coronal FS T2-weighted or PD-weighted sequence, and a T1-weighted sagittal sequence. In healthy patients, the contrast agent surrounds the articular surface of the SSC tendon, and underlines the LHBT in its groove, without any leak of contrast agent in front of the lesser tuberosity, or in the SASD bursa.

\section{Computed tomography arthrography (CTA)}

The same technique of arthrography outlined above is also performed for CTA. Ten to twelve cc of contrast medium are injected into the glenohumeral joint (nonionic iodine agent at 200-300 mgI/mL). Spiral scanning using a small field of view allows isotropic data acquisition and mutiplanar reformation. CTA allows a precise analysis of SSC tendon tears thanks to its high resolution and high positive contrast between the tendon and contrast medium.

\section{Pathological features}

\section{Tendinopathy}

In calcific tendinitis, plain radiographs allow localization and assessment of the texture and morphology of calcific deposits (Fig. 4). Ultrasound examination is also a fundamental tool in the diagnosis of calcific tendinitis. It shows a hypoechoic SSC tendon that loses its fibrillary echo structure. It can also show associated calcification, and specify its topography and morphology. Voluminous calcifications can be responsible for coracoid impingement, which must be investigated using dynam-

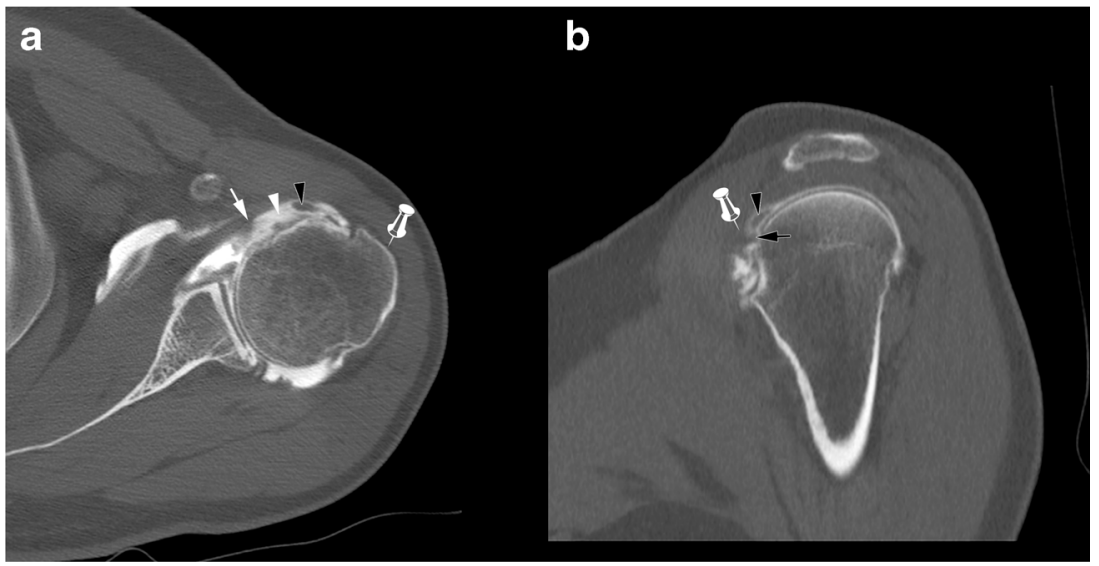

Fig. 7 Forty-five-year old man with pain of the anterior shoulder. Computed tomography arthrography of the left shoulder with axial (a) and sagittal oblique (b) reconstruction. Complete partial thickness tear of the SSC tendon as shown by the absence of opacification of SASD bursa (pin), and the whole disruption of the tendon in the lateral direction (arrow head). The tendinous stump presents an intermediary retraction (arrow). This tear leads to a medial subluxation of the LHBT (black arrowhead) (a). Note that an intact superior glenohumeral ligament (black arrow) prevents the LHBT from inferior dislocation (b) 
Fig. 8 Computed tomography athrography of the left shoulder with axial (a) and sagittal oblique (b) multiplanar reconstructions. Opacification of the SASD (pin) and whole disruption, either laterally (a) or vertically (b) (arrow heads) of the SSC tendon are consistent with a complete full thickness tear. Intermediate retraction of the tendinous stump (arrow)

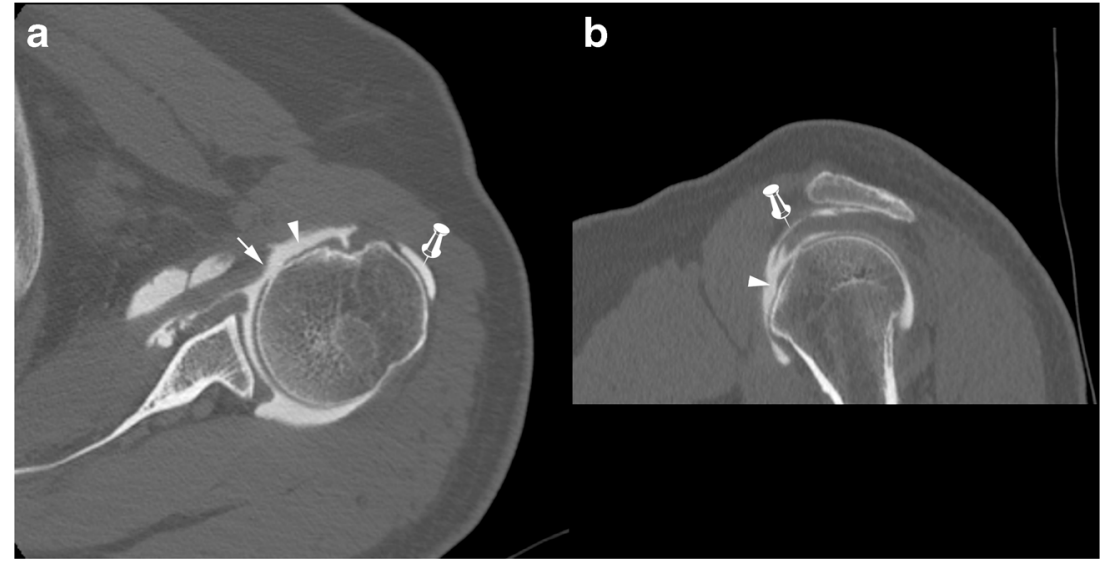

ic study in internal rotation. Increased signal in colour Doppler is correlated with intensity of symptoms [24].

MRI provides no additional information for the evaluation of calcific tendinitis. It shows a non-fluid intermediary high signal on $\mathrm{T} 2$ or $\mathrm{PD}_{\mathrm{w}}$ sequences [25]. Calcifications appear as
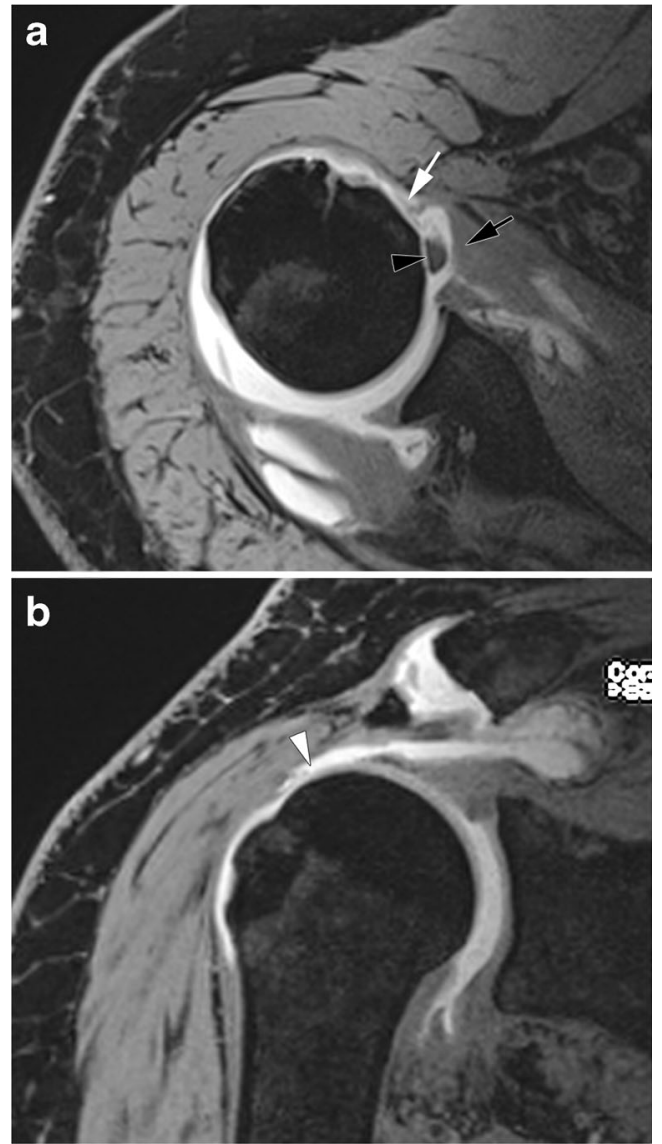

Fig. 9 Axial (a) and sagittal oblique reformats (b) from a 3D FS T1weighted MRA. Massive rotator cuff tear as shown by complete full thickness tears of the SSC (white arrow) (a) and the SSP (white arrow head) tendons (b). LHBT is medially dislocated (black arrow head) (a), and there is a proximal retraction of SSC tendon (black arrow) low signal in all sequences and can therefore be missed. High signal on $\mathrm{T} 2{ }_{\mathrm{w}}$ images around calcifications can be seen because of oedema at the resorptive phase [26].

\section{SSC tendon tears}

Most SSC lesions (Fig. 5) are part of an extended rotator cuff tear. They can also occur in anterosuperior lesions of the rotator cuff, involving the rotator interval, and the SSP, SSC, and LHB tendons. There are $80 \%$ of SSC tendon tears associated with a SSP tear [27]. Isolated SSC tendon tears are uncommon (Fig. 6) and occur in specific mechanisms (traumatism, glenohumeral instability) [28].

These tears are best categorized as full-thickness if communicating with the SASD (partial-thickness if not) and complete if the whole tendon is disrupted (incomplete if not). Most of the time, SSC tendon tears are partial thickness tears involving the articular side of the tendon. On ultrasound, partial thickness tears appear as a hypoechoic tendon, with focal interruption of deep fibres, at the articular side of the tendon [29]. MRI shows a local high signal on FS T2weighted images, without any extension to the bursal surface. The most sensitive sign in CTA and MRA is the presence of contrast agent in front of the lesser tuberosity [28]. Absence of opacification of the SASD bursa rules out a full thickness tear.

Partial thickness tears start at the superomedial aspect of its enthesis and progress inferolaterally [22]. As a consequence, incomplete partial thickness tear (where only the superomedial fibers are disrupted) evolve into complete partial thickness tear (where the whole tendon is disrupted but its superficial aponeurosis and transverse ligament) (Fig. 6). In the latter case, MRA and CTA show a continuity between the intra-articular contrast and the contrast in the bicipital groove (Fig. 7). The SSC tendon can stay in its place, maintained by the transverse ligament, or can be retracted. 
Full thickness tears, whether partial or complete, are less common. Since the transverse ligament is also injured in these cases, there is a communication between the glenohumeral joint and the SASD bursa. In partial full thickness tears, only some fibres of the SSC tendon are injured, whereas complete full thickness tears involve all the SSC tendon fibres. This kind of tear is more frequent when there is a history of trauma, and beyond the age of 40 years [30].

Ultrasound shows a hypoechoic or anechoic defect from the articular to bursal surface of the SSC tendon. MRI shows a fluid high signal on $\mathrm{T} 2{ }_{\mathrm{w}}$ images involving the full thickness of the SSC tendon. Opacification of the SASD bursa is observed on MRA or CTA because of the transfixing tear.

In case of a complete full thickness SSC tendon tear, tendinous stump retraction must be evaluated: it can be located in front of the anatomical neck (intermediate retraction) (Fig. 8) or the glenohumeral joint (proximal retraction) (Fig. 9) [22].

More rarely, there may be partial thickness tears at the bursal side of the tendon (Fig. 10), and/or horizontal cleavage tear of the SSC tendon (lamellar dissection), with the LHBT inserting into the cleavage [22]. It is an example of a hidden lesion, referring to an injury of the biceps pulley whose arthroscopic and clinical diagnosis is challenging (Fig. 11).

The sensitivity and specificity of ultrasound, MRI, CTA, and MRA for SSC lesions are detailed in Table 1.

Injury of the LHBT is associated in $50 \%$ cases of SSC tendon tears [22]. The LHBT can be dislocated (in front of the lesser tuberosity and sometimes into the glenohumeral joint), or sub-dislocated (LHBT located in front of the internal aspect of the bicipital groove) (Fig. 12). The LHBT can also be torn. Indirect ultrasound features of LHBT subdislocation are widening and flattening of the LHBT [22]. Moreover, the anechogenic triangle separating the LHBT from the internal aspect of the bicipital groove is no longer visualized (Brasseur's triangle sign).

Fatty degeneration of the SSC muscle is usually evaluated on CT or MRI (Fig. 13) using Goutallier's grading system [35]. Quantitative measurement of Hounsfield units within the SSC muscle on sagittal CT images correlates well with Goutallier grades and may also be used [36]. Conversely, measurement of signal intensity in MRI is not reliable for the evaluation of fatty degeneration [37]. Experience shows that fatty degeneration is
Fig. 10 Axial FS T1 weighted MRA images $(\mathbf{a}, \mathbf{b}, \mathbf{c})$ and axial oblique reformat through the LHBT (d). The inferior portion of the SSC tendon is normal (white arrow) (a), but there is an incomplete partial tear of the bursal side of the superior portion of the SSC tendon. This is responsible for a medial subluxation of the LHBT (black arrow head). Superiorly, the horizontal portion of the LHBT is hypertrophic (black arrow head), consistent with an "hourglass" biceps
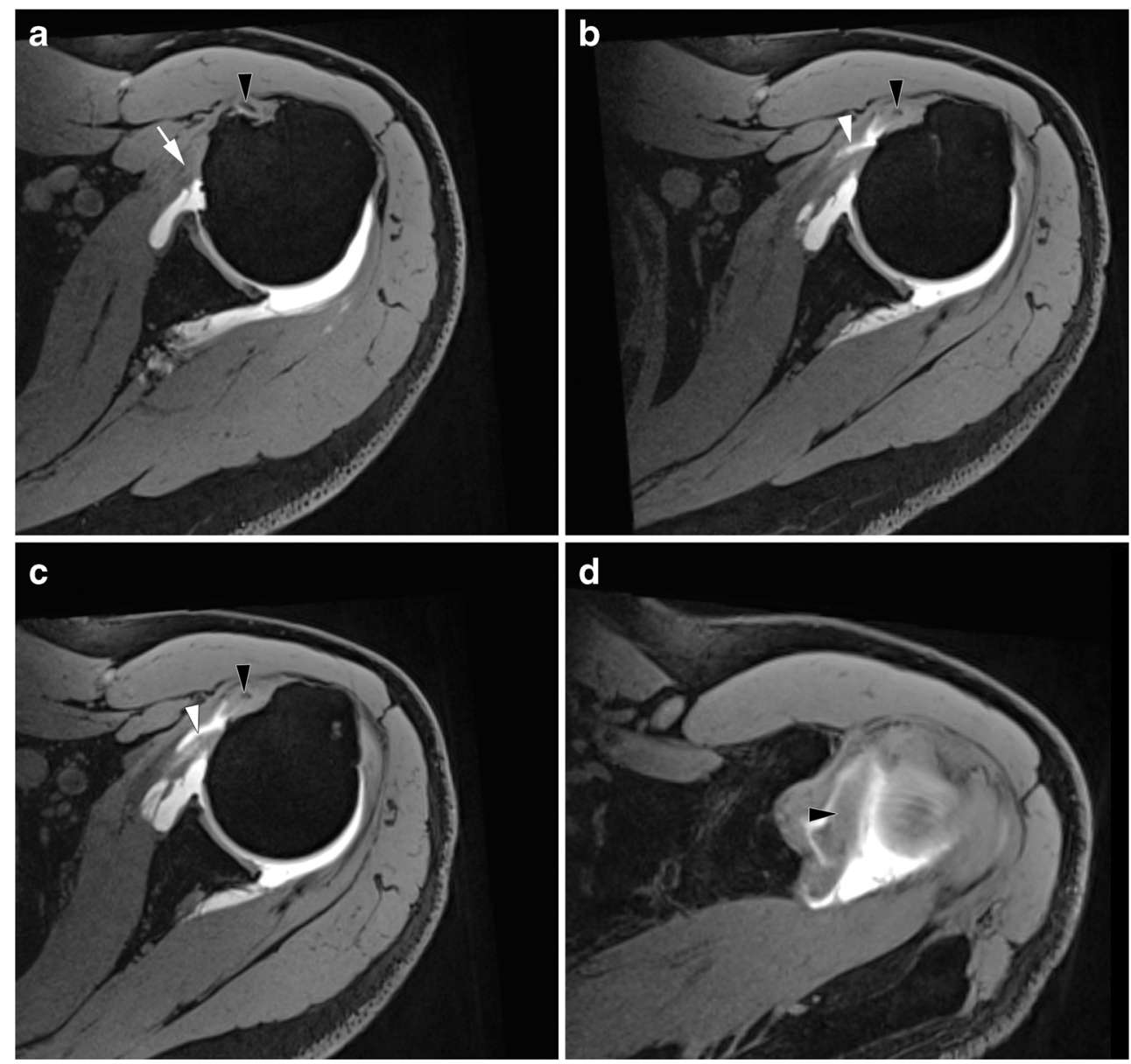

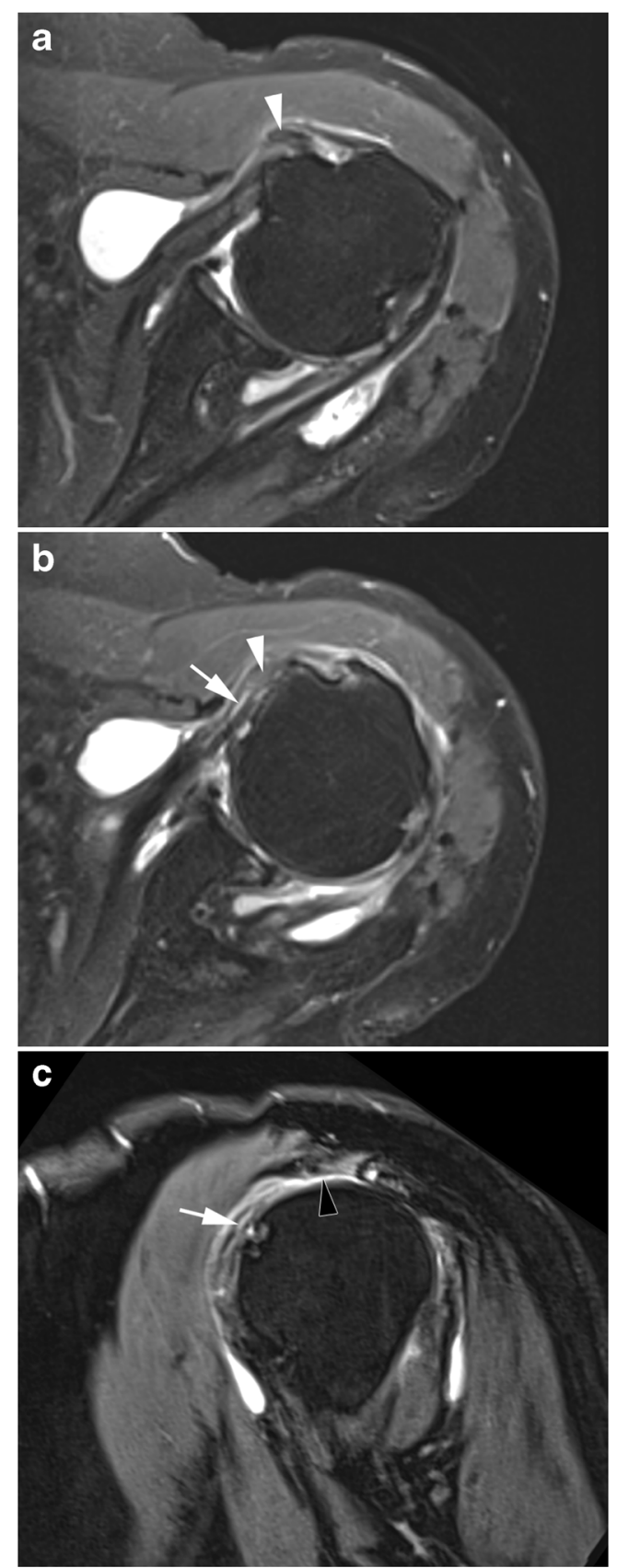

Fig. 11 Left shoulder MRI of a 76-year-old woman. On axial FS PDweighted images, the LHBT (white arrow heads) is medially displaced (a), and incarcerated into a cleavage of the SSC tendon (white arrow (b). On the sagittal oblique FS T2-weighted image (c), the SSC tendon tear (white arrow) is associated with a complete full thickness tear of the SSP tendon (black arrow head)

often greater in the upper part of the SSC muscle, probably because the SSC tendon tears begin superiorly.

There is a significant correlation between Goutallier grades and rupture rate after surgical repair (i.e., rerupture) : while it is $<10 \%$ in grade 0 and 1 , it increases to $28 \%$ in grade 2, and $50 \%$ in grade 3 [4]. Rerupture rate is also correlated with the delay between injury and surgery [4].
Table 1 Sensitivity and specificity of imaging techniques for the diagnosis of SSC tendon tears

\begin{tabular}{llll}
\hline Imaging technique & Sensitivity & Specificity & Reference \\
\hline Ultrasound & $39.5 \%$ & $93.1 \%$ & {$[31]$} \\
MRI & $83 \%$ & $70 \%$ & {$[32]$} \\
MR-arthrography & $81-83 \%$ & $82-83 \%$ & {$[33]$} \\
CT-arthrography & $64.7 \%$ & $98.2 \%$ & {$[34]$} \\
\hline
\end{tabular}

$M R A$ magnetic resonance arthrography, $C T$ computed tomography

In post-traumatic SSC tendon tears, early arthroscopic repair (within an average of 3.7 months) leads to good functional results, a decrease in fatty degeneration and improvement of muscle mass on imaging [38].

\section{SSC tendon lesions and anterior instability}

SSC tendon lesions have been described as a contributing factor to anterior glenohumeral dislocation, and vice versa. When associated with anterior instability, the middle and lower part of SSC tendon are injured [39]. Therefore, when investigating anterior instability, SSC tendon lesions must be investigated, because their repair is part of the treatment of recurrent anterior shoulder dislocations. Repair of the SSC tendon is therefore associated in the Bankart arthroscopic technique with repair of capsular, labral, and ligamentous lesions (Fig. 14a). Another surgical technique for anterior instability treatment, especially when there is associated glenoid bone loss, is the Latarjet procedure (Fig. 14b) (open surgery or arthroscopy) [40]. This procedure involves the removal and transfer of a section of the coracoid process with its attached muscle to the front of the glenoid. The Latarjet mechanism of stability is twofold:

- The coracoid acts as a bone block and increases the joint surface contact area.

- The conjoint tendon centralizes the humeral head into the joint.

\section{Coracoid (antero-medial) impingement}

Coracoid impingement (CI) results from impingement of the SSC tendon or LHBT between the coracoid process and the lesser tuberosity [41]. CI is quite uncommon, described in $5.1 \%$ patients who underwent rotator cuff surgery in Suenaga's study [42], but more frequent (19\%) in cases of extended rotator cuff tear (SSP, SSC and infraspinatus) [43] because of an anterior subdislocation of the humeral head (Fig. 13). Other contributing factors that have been described are: 


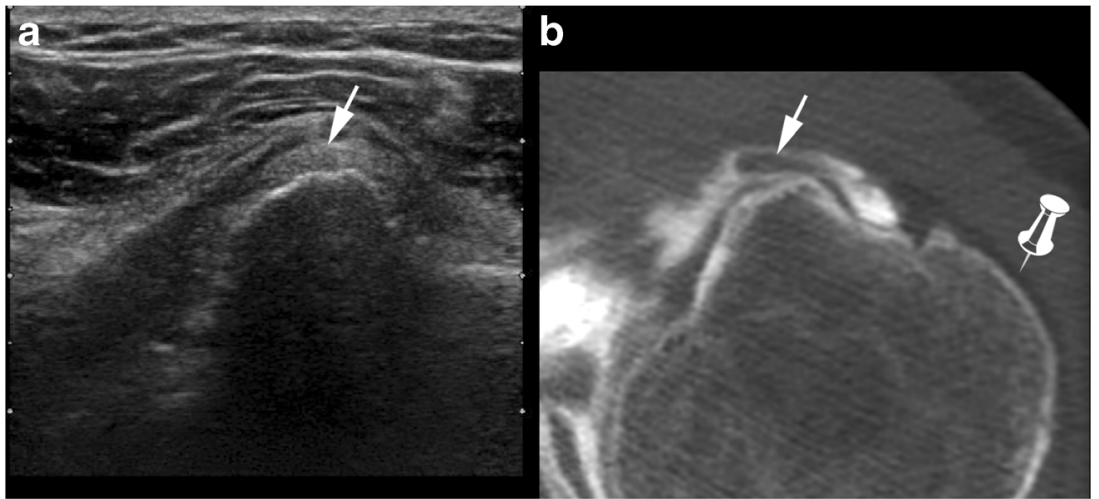

Fig. 12 Ultrasound (a) and computed tomography (CT) arthography (b) correlation: complete partial thickness tear of the upper SSC tendon responsible for subluxation of the LHBT (white arrow), located in front of the medial edge of the intertubercular sulcus. On CT-arthrography (b), contrast in the intertubercular groove is in direct continuity with intraarticular contrast, without any opacification of the SASD bursa (pin) because the bursal side of the transverse ligament is intact
- acquired factors: calcifications or ossification of the SSC tendon, mucoid cyst, tumour of the coracoid process, soft tissue tumour in the subcoracoid space, post traumatic (hypertrophic callus bone after proximal humerus, or coracoid process, or glenoid fractures) [44-47];
- iatrogenic factors: surgery of anterior or posterior instability, acromionectomy;

- constitutional factors: narrowness of the subcoracoid space. There is wide anatomical variability in the size and shape of the coracoid process. A coraco-glenoid
Fig. 13 Sixty-two-year old man with a massive right rotator cuff tear involving the SSP, LHBT and SSC tendons. Transverse US image (a): exposure of the lesser tuberosity (white arrow heads) consistent with a complete full thickness tear of the SSC tendon. The intertubercular sulcus (white arrow) is empty because the LHBT is torn. Axial FS DP weighted MRI image (b): proximal retraction of the torn SSC tendon (black arrow). On sagittal T1 weighted MRI images (c) there is severe amyotrophy and fatty degeneration of the SSC muscle (white outline). On coronal FS DP weighted MRI images, there is an intermediate retraction of the torn SSP tendon (pin) (d). As the SSC tendon can no longer fulfil its stabilizing role, there is passive instability and an anterior sub-dislocation of the humeral head coming in contact with the coracoid process (lightning) (b). Also note an associated tear of the acromial head of the deltoid tendon (black arrow heads) (b, d)
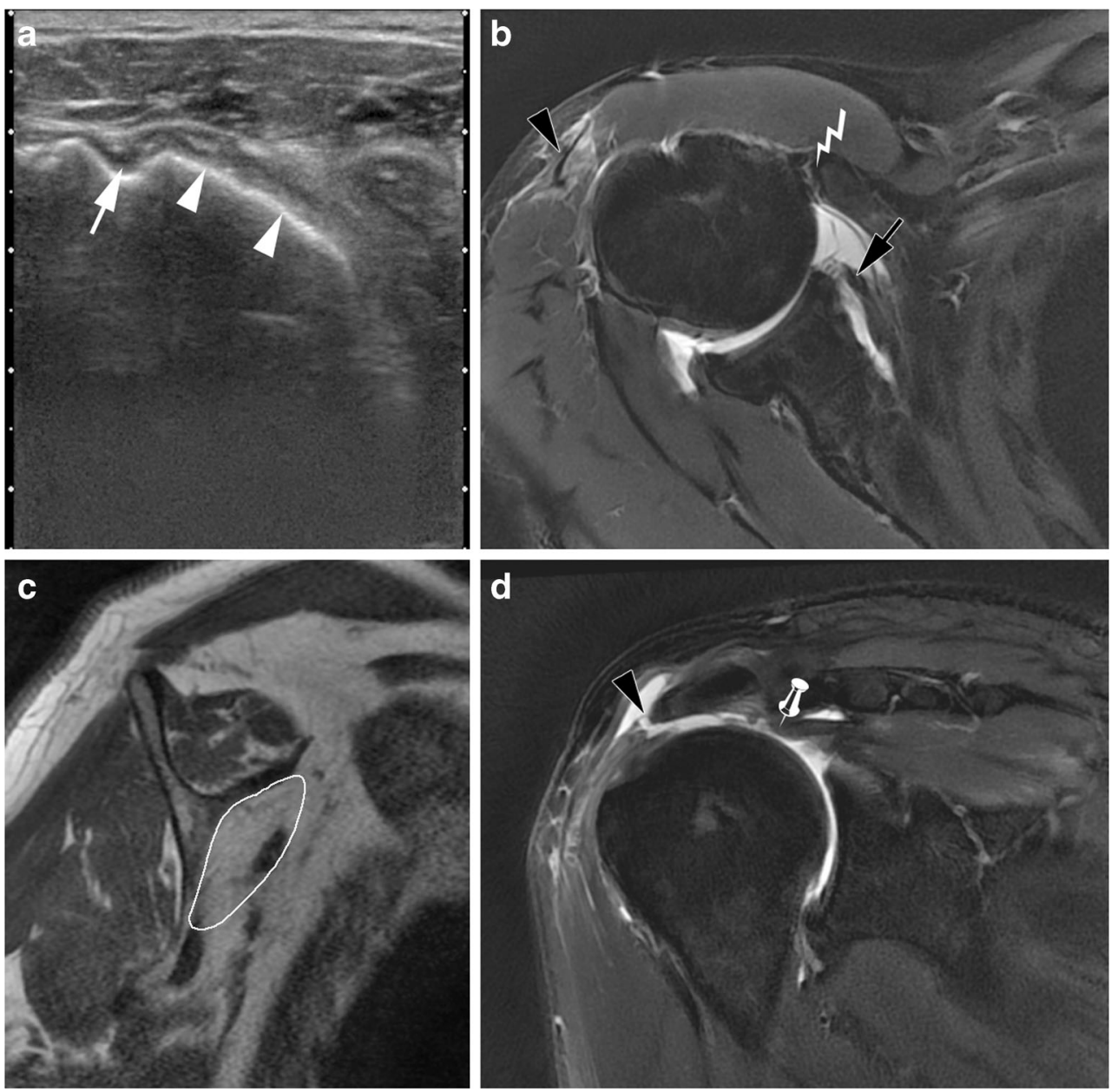
Fig. 14 Schematic

representations of surgical

treatment of shoulder anterior

instability: Bankart (a) and

Latarjet (b) procedures
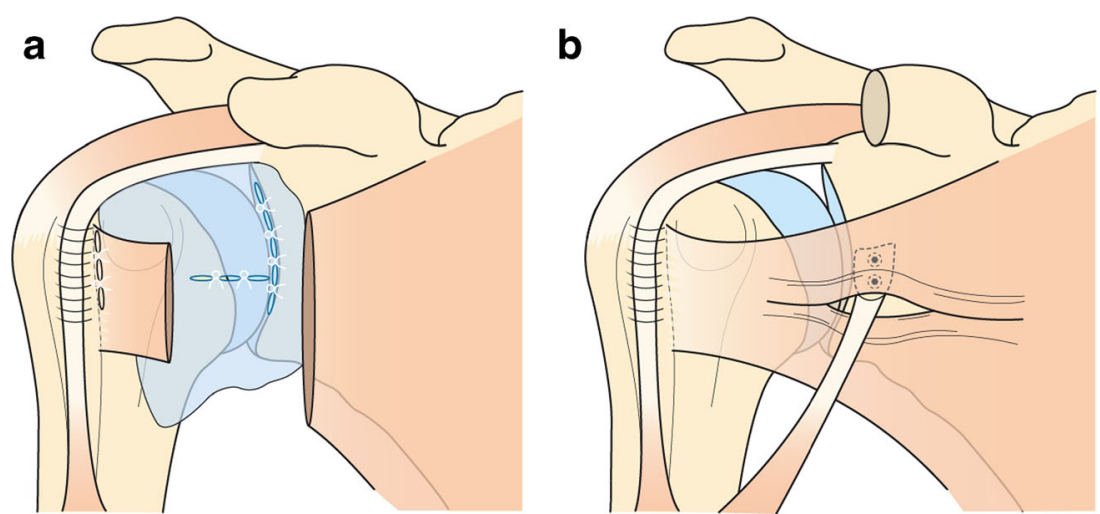

space in the shape of a "round bracket" is associated with a short coraco-humeral distance and could therefore be a predisposing factor to CI syndrome [48]. The coracohumeral distance (CHD) is defined as the shortest distance between the gleno-humeral head and the coracoid process on axial CT or MRI. A CHD $<11 \mathrm{~mm}$ is sensitive but not specific for CI syndrome [49]. Dynamic ultrasound study is interesting, because the CHD decreases in internal rotation and elevation. The values of $\mathrm{CHD}$ associated with CI on ultrasound vary from 6 to $8 \mathrm{~mm}$, according to authors $[50,51]$.

- A direct ultrasound sign of CI is the deformation of the bursal side of the SSC tendon when passing below the coracoid process, which can be associated with a sensation of projection under the probe (Fig. 15). Subcoracoid bursitis is an indirect sign of CI.

An accessory coraco-brachialis muscle is also a constitutional factor of CI, rarely visualized on ultrasound or MRI in front of the SSC [15].

\section{Muscle injury}

Throwing-related intrinsic lesions of the SSC muscle (Fig. 16) have been described, affecting mostly the inferior half of the SSC muscle at the myotendinous junction. These injuries
Fig. 15 Axial dynamic ultrasound images during progressive internal rotation of the shoulder (from a to d). Anatomic landmarks are the coracoid process (white arrow head), the lesser tuberosity (black arrow heads) and the intertubercular sulcus (black arrow). A macrocalcification is seen in the SSC tendon (arrow). It gradually approaches the coracoid process (b and $\mathbf{c}$ ). In internal rotation (d), pain and sensation of projection under the probe when calcification passes under the coracoid process, are consistent with coracoid impingement (lightning) associated with calcific tendinitis
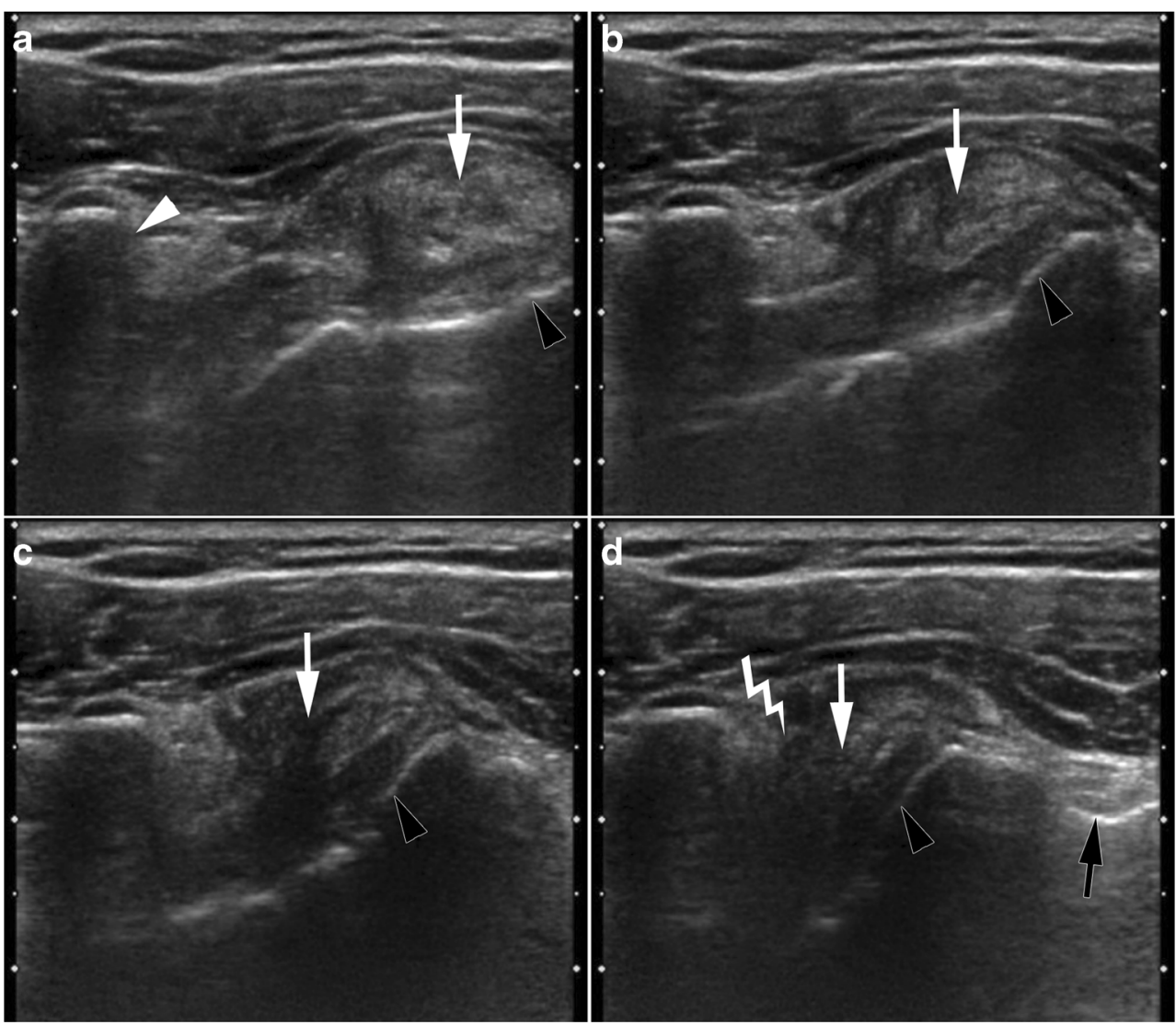
Fig. 16 Right shoulder MRI of a 21-year-old military serviceman with sharp pain of the right shoulder, occurring after military training. Axial (a) and coronal oblique (b) FS DP weighted images. Mottled and linear high signal areas in the SSC muscle mostly at the myotendinous junctions (white arrows) are secondary to intrinsic muscle lesions

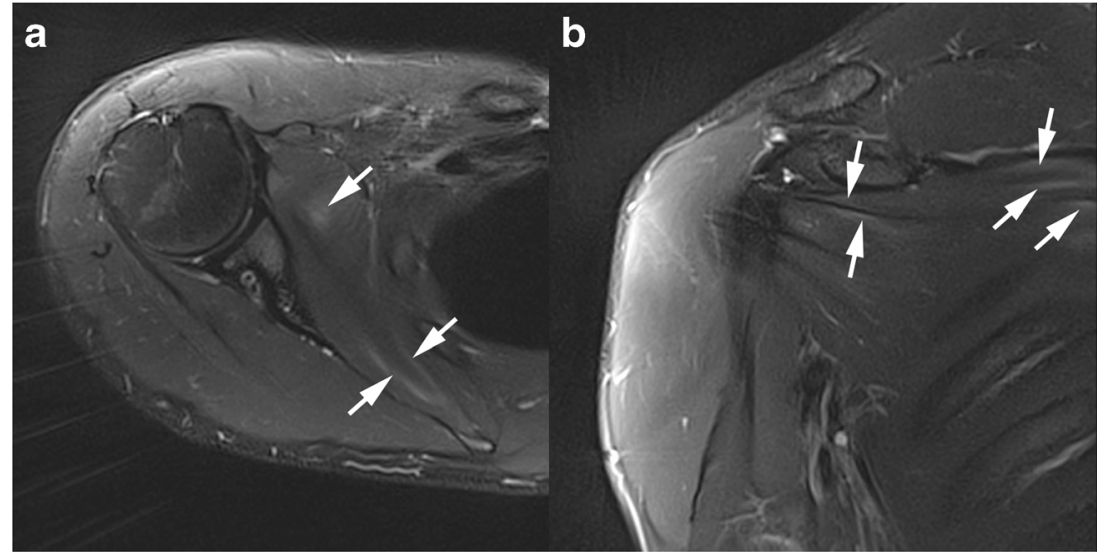

occur mostly in baseball players having lower levels of dominant arm external rotation, leading to greater stretching of the SSC muscle [52].

\section{Conclusion}

Although not well known and less common that SSP tendon lesions, SSC tendon lesions are more common than generally expected. The coupling of radiography with ultrasound yields an accurate diagnosis in most cases. Cross-sectional imaging techniques, especially CTA and MRA, allow accurate characterization of SSC tendon lesions, and identify their muscular impact. These elements are fundamental for the surgeon to adapt the surgical technique. Classically, SSC tendon tears are partial thickness tears starting superomedially and progressing inferolaterally. In most cases, SSC tendon tears are not isolated. Particular mechanisms, such as anterior instability or coracoid impingement, lead to specific injuries of the SSC tendon and specific surgical treatments. Intrinsic muscle injuries at the myotendinous junction have also been described.

Open Access This article is distributed under the terms of the Creative Commons Attribution 4.0 International License (http:// creativecommons.org/licenses/by/4.0/), which permits unrestricted use, distribution, and reproduction in any medium, provided you give appropriate credit to the original author(s) and the source, provide a link to the Creative Commons license, and indicate if changes were made.

\section{References}

1. Ward SR, Hentzen ER, Smallwood LH, Eastlack RK, Burns KA, Fithian DC et al (2006) Rotator cuff muscle architecture: implications for glenohumeral stability. Clin Orthop 448:157-163

2. Morag Y, Jamadar DA, Miller B, Dong Q, Jacobson JA (2011) The subscapularis: anatomy, injury, and imaging. Skeletal Radiol 40(3): 255-269
3. Arai R, Nimura A, Yamaguchi K, Yoshimura H, Sugaya H, Saji T et al (2014) The anatomy of the coracohumeral ligament and its relation to the subscapularis muscle. J Shoulder Elb Surg Am Shoulder Elb Surg Al 23(10):1575-1581

4. Flury MP, John M, Goldhahn J, Schwyzer H-K, Simmen BR (2006) Rupture of the subscapularis tendon (isolated or in combination with supraspinatus tear): when is a repair indicated? J Shoulder Elb Surg Am Shoulder Elb Surg Al 15(6):659-664

5. Wright JM, Heavrin B, Hawkins RJ, Noonan T (2001) Arthroscopic visualization of the subscapularis tendon. Arthrosc $\mathrm{J}$ Arthrosc Relat Surg Off Publ Arthrosc Assoc N Am Int Arthrosc Assoc 17(7):677-684

6. Mansat P, Frankle MA, Cofield RH (2003) Tears in the subscapularis tendon: descriptive analysis and results of surgical repair. Jt Bone Spine Rev Rhum 70(5):342-347

7. Kasper JC, Itamura JM, Tibone JE, Levin SL, Stevanovic MV (2008) Human cadaveric study of subscapularis muscle innervation and guidelines to prevent denervation. J Shoulder Elbow Surg 17(4):659-662

8. O'Connell NE, Cowan J, Christopher T (2006) An investigation into EMG activity in the upper and lower portions of the subscapularis muscle during normal shoulder motion. Physiother Res Int J Res Clin Phys Ther 11(3):148-151

9. Kronberg M, Németh G, Broström LA (1990) Muscle activity and coordination in the normal shoulder. An electromyographic study Clin Orthop 257:76-85

10. Symeonides PP (1972) The significance of the subscapularis muscle in the pathogenesis of recurrent anterior dislocation of the shoulder. J Bone Joint Surg Br 54(3):476-483

11. Halder A, Zobitz ME, Schultz E, An KN (2000) Structural properties of the subscapularis tendon. J Orthop Res Off Publ Orthop Res Soc 18(5):829-834

12. Le Corroller T, Cohen M, Aswad R, Champsaur P (2007) The rotator interval: hidden lesions? J Radiol 88(11 Pt 1):1669-1677

13. Gaskill TR, Braun S, Millett PJ (2011) Multimedia article. The rotator interval: pathology and management. Arthrosc J Arthrosc Relat Surg Off Publ Arthrosc Assoc N Am Int Arthrosc Assoc 27(4):556-567

14. Arai R, Mochizuki T, Yamaguchi K, Sugaya H, Kobayashi M, Nakamura T et al (2010) Functional anatomy of the superior glenohumeral and coracohumeral ligaments and the subscapularis tendon in view of stabilization of the long head of the biceps tendon. J Shoulder Elb Surg Am Shoulder Elb Surg Al 19(1):58-64

15. Bauones S, Moraux A (2015) The accessory coracobrachialis muscle: ultrasound and MR features. Skeletal Radiol 44(9):1273-1278 
16. Milgrom C, Schaffler M, Gilbert S, van Holsbeeck M (1995) Rotator-cuff changes in asymptomatic adults. The effect of age, hand dominance and gender. J Bone Joint Surg Br 77-B(2):296-298

17. Cotten A, Boutry N, Demondion X, Maynou C (2008) Pathologies de la coiffe des rotateurs. In: Imagerie musculosquelttique Pathologies locoregionales. Elsevier Masson

18. Bard H. Echographie de l'épaule. Examen clinique. Gel-Contact. 2004;(13):12-4

19. Gerber C, Krushell RJ (1991) Isolated rupture of the tendon of the subscapularis muscle. Clinical features in 16 cases. J Bone Joint Surg Br 73-B(3):389-394

20. Lin L, Yan H, Xiao J, Ao Y, Cui G (2015) Internal rotation resistance test at abduction and external rotation: a new clinical test for diagnosing subscapularis lesions. Knee Surg Sports Traumatol Arthrosc Off J ESSKA 23(4):1247-1252

21. Wissman RD, Kapur S, Akers J, Crimmins J, Ying J, Laor T (2009) Cysts within and adjacent to the lesser tuberosity and their association with rotator cuff abnormalities. AJR Am J Roentgenol 193(6): 1603-1606

22. Bernageau J, Goutallier D (1997) Isolated lesions of the subscapularis tendon and internal malpositions of the biceps tendon. Apropos of 45 cases]. J Radiol 78(12):1255-1263

23. Lungu E, Moser TP (2015) A practical guide for performing arthrography under fluoroscopic or ultrasound guidance. Insights Imaging 22

24. Chiou H-J, Chou Y-H, Wu J-J, Hsu C-C, Huang D-Y, Chang C-Y (2002) Evaluation of calcific tendonitis of the rotator cuff: role of color Doppler ultrasonography. J Ultrasound Med Off J Am Inst Ultrasound Med 21(3):289-295

25. Yablon CM, Jacobson JA (2015) Rotator cuff and subacromial pathology. Semin Musculoskelet Radiol 19(3):231-242

26. Merolla G, Singh S, Paladini P, Porcellini G (2015) Calcific tendinitis of the rotator cuff: state of the art in diagnosis and treatment. J Orthop Traumatol Off J Ital Soc Orthop Traumatol. 12.

27. Li XX, Schweitzer ME, Bifano JA, Lerman J, Manton GL, ElNoueam KI (1999) MR evaluation of subscapularis tears. J Comput Assist Tomogr 23(5):713-717

28. Pfirrmann CWA, Zanetti M, Weishaupt D, Gerber C, Hodler J (1999) Subscapularis tendon tears: detection and grading at MR arthrography. Radiology 213(3):709-714

29. van Holsbeeck MT, Kolowich PA, Eyler WR, Craig JG, Shirazi KK, Habra GK et al (1995) US depiction of partial-thickness tear of the rotator cuff. Radiology 197(2):443-446

30. Zanetti M, Weishaupt D, Jost B, Gerber C, Hodler J (1999) MR imaging for traumatic tears of the rotator cuff: high prevalence of greater tuberosity fractures and subscapularis tendon tears. AJR Am J Roentgenol 172(2):463-467

31. Narasimhan R, Shamse K, Nash C, Dhingra D, Kennedy S (2015) Prevalence of subscapularis tears and accuracy of shoulder ultrasound in pre-operative diagnosis. Int Orthop

32. Naimark M, Zhang AL, Leon I, Trivellas A, Feeley BT, Ma CB (2016) Clinical, radiographic, and surgical presentation of subscapularis tendon tears: a retrospective analysis of 139 Patients. Arthrosc J Arthrosc Relat Surg Off Publ Arthrosc Assoc $\mathrm{N}$ Am Int Arthrosc Assoc

33. McGarvey C, Harb Z, Smith C, Houghton R, Corbett S, Ajuied A (2016) Diagnosis of rotator cuff tears using 3-Tesla MRI versus 3Tesla MRA: a systematic review and meta-analysis. Skeletal Radiol 45(2):251-261
34. Charousset C, Bellaïche L, Duranthon LD, Grimberg J (2005) Accuracy of CT arthrography in the assessment of tears of the rotator cuff. J Bone Joint Surg Br 87(6):824-828

35. Goutallier D, Postel JM, Bernageau J, Lavau L, Voisin MC (1995) Fatty infiltration of disrupted rotator cuff muscles. Rev Rhum Engl Ed 62(6):415-422

36. Lee E, Choi J-A, Oh JH, Ahn S, Hong SH, Chai JW et al (2013) Fatty degeneration of the rotator cuff muscles on pre- and postoperative CT arthrography (CTA): is the Goutallier grading system reliable? Skeletal Radiol 42(9):1259-1267

37. Zanetti M, Gerber C, Hodler J (1998) Quantitative assessment of the muscles of the rotator cuff with magnetic resonance imaging. Invest Radiol 33(3):163-170

38. Grueninger P, Nikolic N, Schneider J, Lattmann T, Platz A, Chmiel C et al (2014) Arthroscopic repair of traumatic isolated subscapularis tendon lesions (Lafosse Type III or IV): a prospective magnetic resonance imaging-controlled case series with 1 year of follow-up. Arthrosc J Arthrosc Relat Surg Off Publ Arthrosc Assoc N Am Int Arthrosc Assoc 30(6):665-672

39. Gyftopoulos S, Carpenter E, Kazam J, Babb J, Bencardino J (2012) MR imaging of subscapularis tendon injury in the setting of anterior shoulder dislocation. Skeletal Radiol 41(11):1445-1452

40. Young AA, Maia R, Berhouet J, Walch G (2011) Open Latarjet procedure for management of bone loss in anterior instability of the glenohumeral joint. J Shoulder Elbow Surg 20(2):S61-S69

41. Dumontier C, Sautet A, Gagey O, Apoil A (1999) Rotator interval lesions and their relation to coracoid impingement syndrome. J Shoulder Elb Surg Am Shoulder Elb Surg Al 8(2):130-135

42. Suenaga N, Minami A, Kaneda K (2000) Postoperative subcoracoid impingement syndrome in patients with rotator cuff tear. J Shoulder Elb Surg Am Shoulder Elb Surg Al 9(4):275-278

43. Lo IKY, Burkhart SS (2003) Arthroscopic coracoplasty through the rotator interval. Arthrose J Arthrosc Relat Surg Off Publ Arthrosc Assoc N Am Int Arthrosc Assoc 19(6):667-671

44. Arrigoni P, Brady PC, Burkhart SS (2006) Calcific tendonitis of the subscapularis tendon causing subcoracoid stenosis and coracoid impingement. Arthrosc J Arthrosc Relat Surg 22(10):1139-e1

45. Ko JY, Shih CH, Chen WJ, Yamamoto R (1994) Coracoid impingement caused by a ganglion from the subscapularis tendon. A case report. J Bone Jt Surg Am 76(11):1709-1711

46. Cuéllar A, Cuéllar R, Sánchez A, Cuéllar A, Ruiz-Ibán MA (2015) Soft tissue tumour causing coracoid impingement syndrome. Knee Surg Sports Traumatol Arthrosc 23(9):2635-2638

47. Mavrogenis AF, Valencia JD, Romagnoli C, Guerra G, Ruggieri P (2012) Tumors of the coracoid process: clinical evaluation of twenty-one patients. J Shoulder Elbow Surg 21(11):1508-1515

48. Gumina S, Postacchini F, Orsina L, Cinotti G (1999) The morphometry of the coracoid process - its aetiologic role in subcoracoid impingement syndrome. Int Orthop 23(4):198-201

49. Giaroli EL, Major NM, Lemley DE, Lee J (2006) Coracohumeral interval Imaging in subcoracoid impingement syndrome on MRI. Am J Roentgenol 186(1):242-246

50. Richards DP, Burkhart SS, Campbell SE (2005) Relation between narrowed coracohumeral distance and subscapularis tears. Arthrosc J Arthrosc Relat Surg 21(10):1223-1228

51. Tracy MR, Trella TA, Nazarian LN, Tuohy CJ, Williams GR (2010) Sonography of the coracohumeral interval a potential technique for diagnosing coracoid impingement. J Ultrasound Med 29(3):337-341

52. Polster JM, Lynch TS, Bullen JA, Soloff L, Ilaslan H, Subhas N et al (2016) Throwing-related injuries of the subscapularis in professional baseball players. Skeletal Radiol 45(1):41-47 